\title{
The Linear, Nonlinear and Partial Differential Equations are not Fractional Order Differential Equations
}

\author{
Ali Karci \\ Department of Computer Engineering, Inonu University, Turkey
}

Copyright $(\mathcal{C} 2015$ by authors, all rights reserved. Authors agree that this article remains permanently open access under the terms of the Creative Commons Attribution License 4.0 International License

\begin{abstract}
The differential equations were considered as fractional order differential equations in literature. Homotopy Analysis Method was used to obtain analytical solutions of these equations. We applied reverse processes to analytical solutions of some fractional order differential equations, and observed that solutions could not satisfy the corresponding equations. Due to this case, we proposed a new approach for fractional order derivative and it was verified by using this new approach that any differential equations cannot be converted into fractional order differential equations so simply.
\end{abstract}

Keywords Derivatives, Fractional Calculus, Differential Equations, Fractional Order Differential Equations

MSC Classification: 26A33

\section{Introduction}

Differential equations are applied to a large set of problems, due to this case, there are many studies on differential equations $[1,2,3,4,5]$. Some studies are on converting differential equations into fractional order differential equations.

Fractional order derivatives and fractional order differential equations have important roles in rheology, damping laws, diffusion process, etc $[6,7,8,9,10]$. Partial differential equations, nonlinear differential equations and fractional order differential equations require an effective solutions method. HAM is a candidate method for solving all partial differential equations, nonlinear differential equations and fractional order differential equations [11]. Dehghan and his friends tried to solve nonlinear partial differential equations by using HAM [12].

It is known that these solutions should satisfy the corresponding equations. We applied reverse process to equations and we observed that any differential equation cannot be converted into fractional order differential equation by assuming any order as fractional, since those equations are satisfied by the current conditions; changing the conditions will yield new equations.

The deficiencies in those methods in literature are coming from the fractional order derivative definitions used in the many studies. The methods involving deficiencies yield non-consistent results. Due to this case, there is a method proposed for fractional order derivative in Karc1 studies $[15,16]$ and by using this method concludes in whether converting any differential equations to fractional order differential equations is valid or not. We saw that converting any differential equations into fractional order differential equations by changing order is not a valid method, since applications of the proposed method in Karc1's papers illustrated that any differential equations cannot be converted into fractional order differential equations so simply.

This paper is organized as follow. Section 2 described the applications of HAM to some famous differential equations considered as fractional order differential equations. Section 3 included results of converting differential equations into fractional order differential equations. Finally, Section 4 finalized this paper.

\section{HAM and Some Solutions for Fractional Order Differential Equations}

Shijun [11] proposed an analytic method in his Ph.D. dissertation, and it was called as Homotopy Analysis Method (HAM). Dehghan et al [12] used HAM to solve some fractional order differential equations such as Fractional $\mathrm{KdV}$, Fractional $\mathrm{K}(2,2)$, Fractional Burgers and Fractional Cubic Boussnesq Equations.

Dehghan et al [12] solved these equations and we will apply these solutions to related equations whether they satisfy these equations or not.

$$
\begin{aligned}
& D_{t}^{\alpha} u(x, t) \\
& =\frac{1}{\Gamma(n-\alpha)} \int_{0}^{t}(t-\tau)^{n-\alpha-1} u^{(n)}(x, \tau) d \tau, \quad \alpha>0
\end{aligned}
$$




\subsection{Fractional KdV Equation}

The mathematical model of waves on the shallow water surfaces is Korteweg-de Vries equation (KdV equation), and it is a non-linear partial differential equation whose solutions can be exactly and precisely specified. The $\mathrm{KdV}$ equation can be considered as

$$
u_{t}(x, t)+a\left(u^{2}\right)_{x}(x, t)+b u_{x x x}(x, t)=g(x, t)
$$

Some researchers considered this as fractional order differential equation. The fractional $\mathrm{KdV}$ equation $[13,14]$ is the first equation to be handled in this paper. This equation is shown in Eq.1.

$$
D_{t}^{\alpha} u(x, t)+a\left(u^{2}\right)_{x}(x, t)+b u_{x x x}(x, t)=g(x, t)
$$

Assume that $g(x, t)=0, a=-3$ and $b=1$, the equation in Eq. 2 will be obtained.

$$
D_{t}^{\alpha} u(x, t)-3\left(u^{2}\right)_{x}(x, t)+u_{x x x}(x, t)=0
$$

and this equation was solved by Dehghan et al [12] using HAM [11]. The obtained solution is seen in Eq.3.

$$
u(x, t)=\frac{6 x}{1-36 t},|36 \mathrm{t}|<1, u^{2}(x, t)=\frac{36 x^{2}}{(1-36 t)^{2}}
$$

The function symbols $\mathrm{u}$ and $\mathrm{u}(\mathrm{x}, \mathrm{t})$ will be used interchange. The derivatives are

$$
\begin{gathered}
-3\left(u^{2}\right)_{x}=\frac{-216 x}{(1-36 t)^{2}} \\
u_{x}=\frac{6}{(1-36 t)} \Rightarrow u_{x x}=\frac{0}{(1-36 t)}=0, \text { so, } \mathrm{u}_{\mathrm{xxx}}=0
\end{gathered}
$$

The parameters for fractional order derivative are $n=1$ and $\alpha=\frac{1}{2}$

$$
\begin{aligned}
D_{t}^{\alpha} u & =\frac{216 x}{\Gamma(1 / 2)} \int_{0}^{t} \frac{d \tau}{\sqrt{t-\tau}(1-36 \tau)^{2}} \\
& =-\frac{432 \sqrt{t-\tau}}{\Gamma(1 / 2)(1-26 t)^{2}}+\frac{216 x}{\Gamma(1 / 2)} \int_{0}^{t} \frac{2 \sqrt{t-\tau} d \tau}{(1-36 \tau)^{3}} \\
& =\frac{438 x \sqrt{t}}{\Gamma(1 / 2)}
\end{aligned}
$$
Eq.7.

Substituting results in Eq.4, Eq.5 and Eq. 6 into Eq. 2 yields

$$
\frac{438 x \sqrt{t}}{\Gamma(1 / 2)}-\frac{216 x}{(1-36 t)^{2}}+0 \neq 0
$$

This equation is not same as Eq.2. When $\alpha=1$, $D_{t}^{\alpha} u(x, t)=\frac{216 x}{(1-36 t)^{2}}$ and

$$
\begin{aligned}
& D_{t}^{\alpha} u-3\left(u^{2}\right)_{x}+u_{x x x} \\
& =\frac{216 x}{(1-36 t)^{2}}+\frac{-216 x}{(1-36 t)^{2}}+0=0
\end{aligned}
$$

The results obtained in Eq.7 and Eq. 8 demonstrates that any ordinary differential equation, partial differential equation or nonlinear differential equation cannot be converted to fractional order differential equation with the same coefficients and same orders.

\subsection{Fractional $K(2,2)$ Equation}

A family of nonlinear $\mathrm{KdV}$ equations $\mathrm{K}(\mathrm{m}, \mathrm{n})$ is

$$
u_{t}(x, t)+\left(u^{m}\right)_{x}(x, t)+\left(u^{n}\right)_{x x x}(x, t)=0, m>0,1<n \leq 3 .
$$

Some researchers also considered this equation as fractional order differential equations. The fractional order $\mathrm{K}$ $(2,2)$ equation is the next equation to be handled in this paper [13].

$$
\begin{gathered}
D_{t}^{\alpha} u(x, t)+\left(u^{2}\right)_{x}(x, t)+\left(u^{2}\right)_{x x x}(x, t)=0, \\
\mathrm{u}(\mathrm{x}, 0)=\mathrm{x}, \text { and } 0<\alpha \leq 1
\end{gathered}
$$

The solution of Eq.9 was obtained by Mehdi and his friends and the obtained solution is in Eq.10.

$$
u(x, t)=\frac{x}{1+2 t} \text { and } u^{2}(x, t)=\frac{x^{2}}{(1+2 t)^{2}}
$$

The derivatives of these solutions are

$$
\begin{gathered}
\left(u^{2}\right)_{x}=\frac{2 x}{(1+2 t)^{2}} \\
\left(u^{2}\right)_{x x}=\frac{2}{(1+2 t)^{2}}, \text { and finally } \\
\left(u^{2}\right)_{x x x}=\frac{0}{(1+2 t)^{2}}=0
\end{gathered}
$$

The parameters for fractional order derivative are $n=1$ and $\alpha=\frac{1}{2}$

$$
\begin{aligned}
& D_{t}^{\alpha} u=-\frac{2 x}{\Gamma(1 / 2)} \int_{0}^{t} \frac{d \tau}{\sqrt{t-\tau}(1+2 \tau)^{2}} \\
& =-\frac{2}{\Gamma(1 / 2)}\left[\begin{array}{l}
\left.\frac{2 \sqrt{t-\tau}}{(1+2 \tau)^{2}}\right|_{0} ^{t}+\left.\frac{4 \sqrt{t-\tau}}{(1+2 \tau)^{2}}\right|_{0} ^{t} \\
+2 \int_{0}^{t} \frac{d \tau}{\sqrt{t-\tau}(1+2 \tau)^{2}}
\end{array}\right] \\
& =-\frac{12 x \sqrt{t}}{\Gamma(1 / 2)}
\end{aligned}
$$

The results obtained in Eq.11, Eq.12 and Eq.13 are substituted in Eq.9, and the result in Eq.14 is obtained. 


$$
\begin{aligned}
& D_{t}^{\alpha} u+\left(u^{2}\right)_{x}+\left(u^{2}\right)=0 \\
& \Rightarrow-\frac{12 x \sqrt{t}}{\Gamma(1 / 2)}+\frac{2 x}{(1+2 t)^{2}}+0 \neq 0
\end{aligned}
$$

The obtained result is not equal to zero, so, equation is not satisfied. When $\alpha=1, D_{t}^{\alpha} u(x, t)=\frac{-2 x}{(1+2 t)^{2}}$ and

$$
\begin{aligned}
& D_{t}^{\alpha} u+\left(u^{2}\right)_{x}+\left(u^{2}\right)=0 \\
& \Rightarrow \frac{-2 x}{(1+2 t)^{2}}+\frac{2 x}{(1+2 t)^{2}}+0=0
\end{aligned}
$$

The Eq.14 and Eq.15 illustrates that the ordinary differential equation cannot be converted to fractional order differential equation by substituting any term with fractional order derivative term.

\subsection{Fractional Burgers' Equation}

The mathematical model of gas mechanics and traffic flows is Burgers' equation and this equation is a partial differential equation from fluid mechanics. This equation is also considered as fractional order differential equation. The modified $\mathrm{KdV}$ equation [13] is a Burgers' equation

$$
D_{t}^{\alpha} u(x, t)+\frac{1}{2}\left(u^{2}\right)_{x}(x, t)-(u)_{x x}(x, t)=0, \text { and } 0<\alpha \leq 1
$$

The solution of Eq.16 was obtained by Mehdi and his friends as

$$
u(x, t)=\frac{x}{1+t} \text { and } u^{2}(x, t)=\frac{x^{2}}{(1+t)^{2}}
$$

The derivatives of these solutions are

$$
\begin{array}{r}
\left(u^{2}\right)_{x}=\frac{2 x}{(1+t)^{2}} \\
u_{x}=\frac{1}{1+t} \Rightarrow u_{x x}=\frac{0}{1+t}=0
\end{array}
$$

The parameters are $\mathrm{n}=1$ and $\alpha=\frac{1}{2}$

$$
\begin{aligned}
D_{t}^{\alpha} u & =-\frac{2 x}{\Gamma(1 / 2)} \int_{0}^{t} \frac{d \tau}{(\sqrt{t-\tau})(1+\tau)^{2}} \\
& =\frac{4 x \sqrt{t}}{\Gamma(1 / 2)}
\end{aligned}
$$

The results obtained in Eq.18, Eq.19 and Eq.20 are substituted in Eq.16, and the result in Eq.21 is obtained.

$$
\begin{aligned}
& D_{t}^{\alpha} u+\frac{1}{2}\left(u^{2}\right)_{x}-(u)_{x x}=0 \\
& \Rightarrow \frac{4 x \sqrt{t}}{\Gamma(1 / 2)}+\frac{x}{(1+t)^{2}}-0 \neq 0
\end{aligned}
$$

$$
\begin{gathered}
\text { When } \alpha=1, D_{t}^{\alpha} u(x, t)=\frac{-x}{(1+t)^{2}} \text { and } \\
D_{t}^{\alpha} u+\frac{1}{2}\left(u^{2}\right)_{x}-(u)_{x x}=0 \\
\Rightarrow \frac{-x}{(1+t)^{2}}+\frac{x}{(1+t)^{2}}-0=0
\end{gathered}
$$

The Eq.21 and Eq.22 illustrates that the ordinary differential equation cannot be converted to fractional order differential equation by substituting any term with fractional order derivative term.

\subsection{Fractional Cubic Boussinesq Equation}

The original Boussinesq equation is $u_{t t}(x, t)-u_{x x}(x, t)+2\left(u^{3}(x, t)\right)_{x x}-u_{x x x x}(x, t)=0, \quad$ and $\quad$ its fractional order is $D_{t}^{2 \alpha} u(x, t)-u_{x x}(x, t)+2\left(u^{3}(x, t)\right)_{x x}-u_{x x x x}(x, t)=0$ (23)

The solution of Eq.23 was obtained by Mehdi and his friends as

$$
u(x, t)=\frac{1}{x+t} \text { and } u^{3}(x, t)=\frac{1}{(x+t)^{3}}
$$

The derivatives of these solutions are

$$
\begin{gathered}
u_{x}=\frac{-1}{(x+t)^{2}} \Rightarrow u_{x x}=\frac{2}{(x+t)^{3}} \\
u_{x x x}=\frac{-6}{(x+t)^{4}} \Rightarrow u_{x x x x}=\frac{24}{(x+t)^{5}} \\
\left(u^{3}\right)_{x}=\frac{-3}{(x+t)^{4}} \Rightarrow\left(u^{3}\right)_{x x}=\frac{12}{(x+t)^{5}}
\end{gathered}
$$

The parameters are $\mathrm{n}=1$ and $\alpha=\frac{1}{3}$

$$
\begin{aligned}
D_{t}^{2 \alpha} u & =-\frac{1}{\Gamma(1 / 3)} \int_{0}^{t} \frac{(t-\tau)^{-2 / 3} d \tau}{(x+\tau)^{2}} \\
& =\frac{7}{15 \Gamma(1 / 3)} \frac{t^{-2 / 3}}{x}
\end{aligned}
$$

The results obtained in Eq.25, Eq.26, Eq.27 and Eq.28 are substituted in Eq.23, and the result in Eq.29 is obtained.

$$
\begin{aligned}
& D_{t}^{2 \alpha} u-u_{x x}+2\left(u^{3}\right)_{x x}-u_{x x x x}=0 \\
& \Rightarrow \frac{7}{15 \Gamma(1 / 3)} \frac{t^{-2 / 3}}{x}-\frac{2}{(x+t)^{3}} \\
& +\frac{24}{(x+t)^{5}}-\frac{24}{(x+t)^{5}} \\
& \Rightarrow \frac{7}{15 \Gamma(1 / 3)} \frac{t^{-2 / 3}}{x}-\frac{2}{(x+t)^{3}} \neq 0
\end{aligned}
$$

When $\alpha=1$, 


$$
\begin{aligned}
& D_{t}^{2 \alpha} u(x, t)=D_{t}^{\alpha}\left(D_{t}^{\alpha} u(x, t)\right)=\frac{2}{(x+t)^{3}} \text { and } \\
& D_{t}^{2 \alpha} u-u_{x x}+2\left(u^{3}\right)_{x x}-u_{x x x x}=0 \\
& \Rightarrow \frac{2}{(x+t)^{3}}-\frac{2}{(x+t)^{3}}+\frac{24}{(x+t)^{5}}-\frac{24}{(x+t)^{5}}=0
\end{aligned}
$$

The Eq.29 and Eq.30 illustrates that the ordinary differential equation cannot be converted to fractional order differential equation by substituting any term with fractional order derivative term.

\section{Conversion of Differential Equations to Fractional Order Differential Equations}

The sections 2.1, 2.2, 2.3 and 2.4 illustrate that any differential equations cannot be converted into fractional order differential equations easily. The solutions of Fractional KdV, Fractional K(2,2), Fractional Burgers' and Fractional Cubic Boussinesq differential equations by using Homotopy Analyse Method demonstrate that differential equations cannot be converted into fractional order differential equations. There will be two important reasons for this case.

Case 1. Assume that $x^{n}+y^{n}=z^{n}, n, x, y, z \in Z^{+}$. When $n=1$ or 2 there are solutions for this equations, on the other hand, when $\mathrm{n}>2$ there is no solution for this equation. $\mathrm{n}$ can be considered as order of differential equations, and the orders of differential equations are all integers. So, any differential equations cannot be converted into fractional order differential equations without changing the domain of variables of functions.

Case 2. The second reason is that deficiencies in definitions of fractional derivative. Instead of definitions for fractional order derivative, a new definition for fractional order derivative $[15,16]$ can be used to demonstrate that differential equations cannot be converted into fractional order differential equations.

Definition $[15,16]$. Assume that $f(x): R \rightarrow R$ is a function, $\alpha \in R$ and $L($.) be a L'Hospital process. The fractional order derivative of $f(x)$ can be rephrased as follow

$$
\begin{aligned}
& f^{(\alpha)}(x)=\lim _{h \rightarrow 0} L\left(\frac{f^{\alpha}(x+h)-f^{\alpha}(x)}{(x+h)^{\alpha}-x^{\alpha}}\right) \\
& =\lim _{h \rightarrow 0} \frac{\frac{d\left(f^{\alpha}(x+h)-f^{\alpha}(x)\right)}{d h}}{\frac{d\left((x+h)^{\alpha}-x^{\alpha}\right)}{d h}}
\end{aligned}
$$

where $\alpha$ is the order of differentiation.

The effect of this definition can be illustrated on the Fractional KdV Equation, Fractional $\mathrm{K}(2,2)$ Equation, Fractional Burgers' Equation and Fractional Cubic Boussinesq Equation. a) Fractional KdV Equation:

$$
\begin{aligned}
& D_{t}^{\alpha} u(x, t)-3\left(u^{2}\right)_{x}(x, t)+u_{x x x}(x, t)=0 \text { and its solution } \\
& \text { is } u(x, t)=\frac{6 x}{1-36 t},|36 \mathrm{t}|<1 .
\end{aligned}
$$

The fractional order derivatives with respect to new definition (Eq.31) are

$$
\begin{gathered}
D_{t}^{\alpha} u(x, t):\left(\frac{6 x}{t(1-36 t)}\right)^{\alpha-1} \frac{216 x}{(1-36 t)^{2}} \\
-3\left(u^{2}\right)_{x}(x, t):-\frac{216 x}{(1-36 t)^{2}} \\
u_{x}(x, t): \frac{6}{1-36 t}=\frac{6}{(1-36 t)} \\
u_{x x}(x, t)=0 \text { so } u_{x x x}(x, t)=0 .
\end{gathered}
$$

The obtained derivatives are substituted in differential equation (Eq.2) and the obtained result is

$$
\begin{gathered}
D_{t}^{\alpha} u(x, t)-3\left(u^{2}\right)_{x}(x, t)+u_{x x x}(x, t)=0 \\
\left(\frac{6 x}{t(1-36 t)}\right)^{\alpha-1} \frac{216 x}{(1-36 t)^{2}}-\frac{216 x}{(1-36 t)^{2}}+0=0 \\
\Rightarrow\left(\frac{6 x}{t(1-36 t)}\right)^{\alpha-1} \frac{216 x}{(1-36 t)^{2}}=\frac{216 x}{(1-36 t)^{2}} \\
\Rightarrow\left(\frac{6 x}{t(1-36 t)}\right)^{\alpha-1}=1
\end{gathered}
$$

In order to satisfy this equation, $\alpha=1$. If $\alpha=1$, the differential equation (Eq.2) is not a fractional order differential equation.

b) Fractional $\mathrm{K}(2,2)$ Equation:

$$
\begin{aligned}
& D_{t}^{\alpha} u(x, t)+\left(u^{2}\right)_{x}(x, t)+\left(u^{2}\right)_{x x x}(x, t)=0 \quad \text { and } \quad \text { its } \\
& \text { solution is } u(x, t)=\frac{x}{1+2 t} .
\end{aligned}
$$

The fractional order derivatives with respect to new definition (Eq.31) are

$$
\begin{gathered}
D_{t}^{\alpha} u(x, t):-\left(\frac{x}{t(1+2 t)}\right)^{\alpha-1} \frac{2 x}{(1+2 t)^{2}}=\frac{-2 x^{\alpha}}{t^{\alpha-1}(1+2 t)^{\alpha+1}} \\
\left(u^{2}\right)_{x}(x, t): \frac{2 x}{(1+2 t)^{2}} \\
\left(u^{2}\right)_{x x}(x, t): \frac{2}{(1+2 t)^{2}} \\
\left(u^{2}\right)_{x x x}(x, t)=0
\end{gathered}
$$

The obtained derivatives are substituted in differential equation (Eq.9) and the obtained result is 


$$
\begin{aligned}
& D_{t}^{\alpha} u(x, t)+\left(u^{2}\right)_{x}(x, t)+\left(u^{2}\right)_{x x x}(x, t)=0 \\
& \frac{-2 x^{\alpha}}{t^{\alpha-1}(1+2 t)^{\alpha+1}}+\frac{2 x}{(1+2 t)^{2}}+0=0 \Rightarrow \alpha=1 .
\end{aligned}
$$

In order to satisfy this equation, $\alpha=1$. This means that Fractional $\mathrm{K}(2,2)$ Equation (Eq.9) is not a fractional order differential equation.

c) Fractional Burgers' Equation:

$$
\begin{aligned}
& D_{t}^{\alpha} u(x, t)+\frac{1}{2}\left(u^{2}\right)_{x}(x, t)-(u)_{x x}(x, t)=0 \quad \text { and its } \\
& \text { solution is } u(x, t)=\frac{x}{1+t} \text {. }
\end{aligned}
$$

The fractional order derivatives with respect to new definition (Eq.31) are

$$
\begin{gathered}
D_{t}^{\alpha} u(x, t):\left(\frac{x}{t(1+t)}\right)^{\alpha-1} \frac{-x}{(1+t)^{2}}=-\frac{x^{\alpha}}{(1+t)^{\alpha+1}} \\
\frac{1}{2}\left(u^{2}\right)_{x}(x, t): \frac{2 x}{2(1+x)^{2}}=\frac{x}{(1+x)^{2}} \\
(u)_{x}(x, t)=\frac{1}{1+t} \\
-(u)_{x x}(x, t)=0
\end{gathered}
$$

The obtained derivatives are substituted in differential equation (Eq.16) and the obtained result is

$$
\begin{aligned}
& D_{t}^{\alpha} u(x, t)+\frac{1}{2}\left(u^{2}\right)_{x}(x, t)-(u)_{x x}(x, t)=0 \\
& -\frac{x^{\alpha}}{(1+t)^{\alpha+1}}+\frac{x}{(1+t)^{2}}+0=0 \\
& \Rightarrow \frac{x^{\alpha}}{(1+t)^{\alpha+1}}=\frac{x}{(1+t)^{2}} \Rightarrow \alpha=1 .
\end{aligned}
$$

This means that Fractional Burgers' Equation (Eq.16) is not a fractional order differential equation.

d) Fractional Cubic Boussinesq Equation:

$$
\begin{gathered}
D_{t}^{2 \alpha} u-u_{x x}+2\left(u^{3}\right)_{x x}-u_{x x x x}=0 \text { and its solution is } \\
u(x, t)=\frac{1}{x+t} \cdot \\
D_{t}^{2 \alpha} u(x, t):\left(\frac{1}{t(x+t)}\right)^{2 \alpha-1} \frac{-1}{(x+t)^{2}}=-\frac{1}{t^{2 \alpha-1}(x+t)^{2 \alpha+1}} \\
-(u)_{x x}(x, t):-\frac{2}{(x+t)^{3}} \\
2\left(u^{3}\right)_{x x}(x, t)=\frac{24}{(x+t)^{5}} \\
-(u)_{x x x}(x, t)=\frac{6}{(x+t)^{4}}
\end{gathered}
$$

The obtained derivatives are substituted in differential equation (Eq.16) and the obtained result is

$$
\begin{aligned}
& D_{t}^{2 \alpha} u-u_{x x}+2\left(u^{3}\right)_{x x}-u_{x x x x}=0 \\
& \Rightarrow-\frac{1}{t^{2 \alpha-1}(x+t)^{2 \alpha+1}}-\frac{2}{(x+t)^{3}}+\frac{24}{(x+t)^{5}}+\frac{6}{(x+t)^{4}} \neq 0
\end{aligned}
$$

There is no specific $\alpha$ value to satisfy this equation, so, Fractional Cubic Boussinesq Equation is not a fractional order differential equation.

\section{Conclusions}

This paper illustrated that there are important points for differential equations. These points can be listed as below.

a) HAM can be used to solve fractional order differential equations. The obtained solution can be differentiated and result must be substituted in fractional order differential equations. This process should satisfy the equation.

b) Dehghan et al issued this case for some differential equations, and they applied HAM to some differential equations and obtained their solutions. They also assumed that these differential equations can be considered as fractional order differential equations.

c) We applied fractional order derivative method to these solutions and substituted into corresponding equations. This process did not satisfy the corresponding equations. These means that any differential equation cannot be considered as fractional order differential equations so simply.

d) We applied the proposed method in Karc1's papers $[15,16]$ to illustrate that the differentiation order must be equal to 1 for any differential equations, since these equations were obtained by applying differentiation of order 1.

\section{REFERENCES}

[1] F. Schwarz, "Loewy Decomposition of Linear Differential Equations", Bulletin of Mathematical Sciences, vol.3, pp:19-71, 2013.

[2] M.K. Yıldız, B. Karpuz, Ö. Öcalan, "Oscillation of Nonlinear Neutral Delay Differential Equations of Second-Order with Positive and Negative Coefficients", Turkish Journal of Mathematics, vol.33, pp:341-350, 2009.

[3] A. Aghajani, V. Roomi, "An Oscillation Theorem for Second-Order Nonlinear Differential Equations of Euler Type", vol.36, pp:273-280, 2012.

[4] R.Z. Gumbataliev, "On Completeness of Elementary Generalized Solutions of a Class of Operator-Differential Equations of a Higher Order", Turkish Journal of Mathematics, vol.33, pp:383-396, 2009.

[5] M. Nadjafikhah, R.B. Chamazkoti, "Cartan Equivalence Problem for Third-Order Differential Operators", Turkish 
Journal of Mathematics, vol.37, pp:949-958, 2013.

[6] M.J. Ablowitz, P.A. Clarkson, "Solitons, Nonlinear Evolution Equations and Inverse Scattering", Cambridge University Press, New York, 1991.

[7] K.S. Miller, B. Ross, "An Introduction to the Fractional Calculus and Fractional Differential Equations", Wiley, New York, 1993.

[8] I. Podlubny, "Fractional Differential Equations: An Introduction to Fractional Derivatives, Fractional Differentials Equations, to Methods of Their Solution and Some of Their Applications", Academic Press, New York, 1999.

[9] S.G. Samko, A.A. Kilbas, O.I. Marichev, "Fractional Integrals and Derivatives: Theory and Applications", Yverdon, Gordon and Breach, 1993.

[10] B.J. West, M. Bolognab, P. Grigolini, "Physics of Fractal Operators”, Springer, New York, 2003.

[11] L. Shijun, "Homotopy Analysis Method: A New Analytic Method for Nonlinear Problems", Applied Mathematics and
Mechanics, vol.19, pp:957-962,1998.

[12] M. Dehghan, J. Manafian, A. Saadatmandi, "Solving Nonlinear Fractional Partial Differential Equations Using the Homotopy Analysis Method", Numerical Methods for Partial Differential Equations, DOI:10.1002/num.

[13] A.M. Wazwaz, "The Variational Iteration Method for Rational Solutions for K(2,2), Burgers, and Cubic Boussinesq Equations", Journal of Computing and Applied Mathematics, vol.207, pp:18-23, 2007.

[14] D. Kaya, I.E. Inan, "Exact and Numerical Traveling Wave Solutions for Nonlinear Coupled Equations Using Symbolic Computation", Applied Mathematics and Computation, vol.151, pp:775-787, 2004.

[15] A. Karc1, A. Karadoğan, "Fractional Order Derivative And Relationship Between Derivative And Complex Functions", Mathematical Sciences and Applications E-Notes, vol.2, pp:44-54, 2014.

[16] A. Karc1, “A New Approach For Fractional Order Derivative And Its Applications", Universal Journal of Engineering Science, vol.1, pp:110-117, 2013. 